SHORT COMMUNICATION

\title{
CONSTRUCTION AND OPERATION OF A DISPERSIVE LASER RAMAN SPECTROGRAPH USING INTERFERENCE FILTER
}

\author{
K.M. ABEDIN, S.F.U. FARHAD ${ }^{1}$, M.R. ISLAM, AMINUL I. TALUKDER \\ AND \\ A.F.M.Y. HAIDER ${ }^{2}$ \\ Department of Physics, University of Dhaka, Dhaka-1000, Bangladesh
}

\begin{abstract}
A dispersive laser Raman system was designed and constructed using a helium-neon (He-Ne) laser as an excitation source, and an interference filter in the reflection mode for Raleigh filtering instead of the more common holographic notch filter. A commercially available spectrograph equipped with a cooled CCD camera was used to acquire the Raman spectra. The constructed laser Raman spectrograph was found to have excellent performance and sensitivity. Stokes Raman spectra of some common chemicals were acquired by the system, and the wavelengths of spectral lines agreed well with the literature values, within experimental error. The useful spectral range of the system is about $200-4000 \mathrm{~cm}^{-1}$. It was also possible to acquire anti-Stokes Raman spectra of one chemical $\left(\mathrm{CCl}_{4}\right)$ without much difficulty. We hope to use the system for chemical identification of molecules as well as quantitative chemical analysis. To our knowledge, this is the first laser Raman system constructed in Bangladesh.
\end{abstract}

\section{INTRODUCTION}

Raman effect was discovered by C.V. Raman in $1928{ }^{(1)}$ when he observed weak radiation at longer wavelengths when filtered sunlight was used to illuminate a liquid. It was soon found that the frequency shifts were characteristic of the molecules in the liquid, and thus the Raman spectra could be used to identify chemicals. Nowadays, Raman spectroscopy is widely used for many different applications including analytical chemistry, material science, biochemical, medical-and forensic applications. ${ }^{(2-3)}$

Initially, mercury arc lamps, ${ }^{(4)}$ and later, lasers ${ }^{(5-6)}$ were used as sources of monochromatic radiation in Raman spectroscopy. Since Rayleigh scattering of the very strong excitation light tends to mask the weak Raman lines, double and triple monochromators were often used ${ }^{(7-8)}$ to effectively eliminate the Rayleigh-scattered light in Raman spectroscopy. Since 1992, holographic notch filters, ${ }^{(9)}$ having a very high attenuation of the Rayleigh line, has been increasingly used for Rayleigh filtering in a laser Raman system. A holographic notch filter works only at a certain wavelength and is an expensive and relatively rare item, costing more than 500 dollars each.

In this work, we used a conventional interference filter in the reflection mode for Rayleigh filtering instead of the notch filter in conjunction with a dispersive spectrograph to construct a laser Raman spectrographic system. We describe its design, construction and operation, and present the results of measurements performed by it.

\footnotetext{
${ }^{1}$ Present address: Industrial Physics Division, BCSIR Laboratories,Dhaka-1205 Bangladesh.

${ }^{2}$ Author for correspondence.
} 


\section{EXPERIMENTAL}

The schematic diagram of the laser Raman system is shown in Fig. 1. Monochromatic light (at $\lambda=632.8 \mathrm{~nm}$ ) from a $20 \mathrm{~mW}$ unpolarized helium-neon laser (Uniphase) is focused by a short-focus ( $\mathrm{f}=50 \mathrm{~mm}$ ) lens $\mathrm{L} 1$ on the liquid sample kept in a

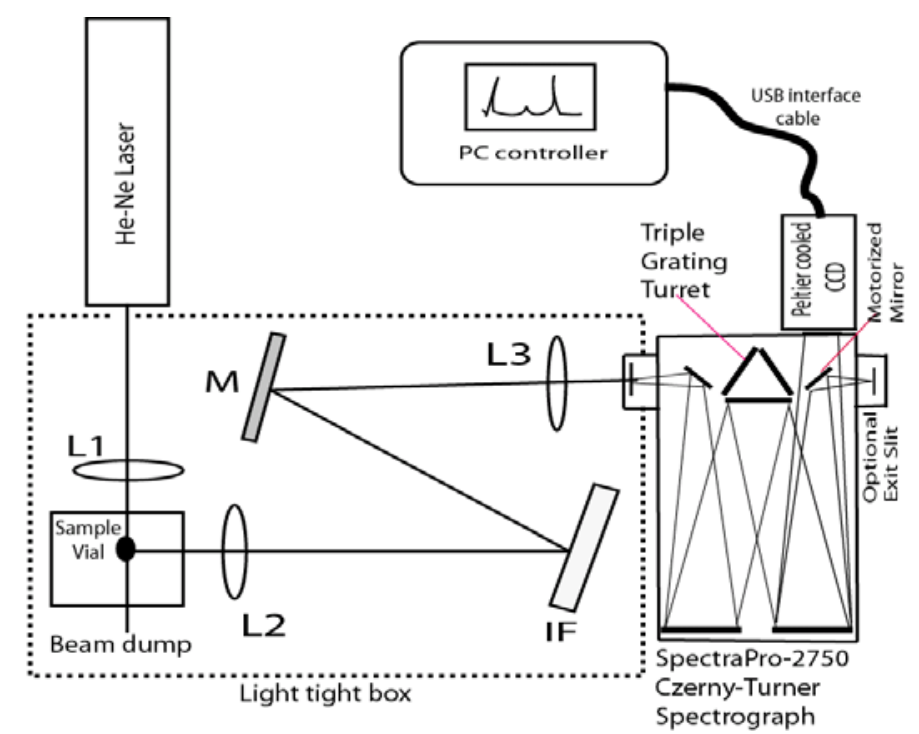

Fig. 1. Schematic diagram of the constructed Raman system. IF is the interference filter, L2 is the collection lens, L3 is the focusing lens.

suitable sample holder (quartz or borosilicate glass bottles). Light scattered at 90 degrees to the incident laser beam was collected by another short-focal length lens L2 ( $\mathrm{f}=50$ $\mathrm{mm}, \mathrm{d}=50 \mathrm{~mm}$ ) and collimated. The light, which contains Rayleigh as well as Raman scattered light, is incident on the 3-cavity dielectric interference filter IF (diameter 25 $\mathrm{mm}$, Coherent). The filter has a peak transmission of $80 \%$ at $632.8 \mathrm{~nm}$ and a FWHM of about $6 \mathrm{~nm}$. As a result of multiple interference inside the filter, light in a narrow band of wavelengths near $632.8 \mathrm{~nm}$ (Rayleigh-scattered light) is mostly transmitted through it, while light outside this band is mostly reflected. The reflected light, containing the Raman-scattered energy, is directed by a mirror $\mathrm{M}$ towards the spectrograph input. The light is focused on the input slit of the spectrograph by the lens L3 ( $\mathrm{f}=75 \mathrm{~mm})$. The entire optics on the input side is inside a light-tight box in order to reduce the effect of stray light which can interfere with the sensitive Raman measurements. The box has a door on one side and by opening it sample vial can be placed in the system for examination.

The spectrograph (Acton 2750SP) is a Czerny-Turner system using an interchangeable 3-grating turret and has a focal length of $750 \mathrm{~mm}$. The input side of the spectrograph has an adjustable slit. The resolution of the system with a 300 lines $/ \mathrm{mm}$ diffraction grating and 10 micron slit width was about $0.1 \mathrm{~nm}$ (about $2.5 \mathrm{~cm}^{-1}$ ). The 
diffracted light falls on a deep-cooled CCD camera (Princeton PIXIS 100B) attached to the output port. The camera has a $1340 \times 100$ pixel CCD elements cooled to $-75^{\circ} \mathrm{C}$ by Peltier coolers in order to reduce dark noise. The acquired data (spectrum) can be transferred to the PC by a USB cable, and all camera functions, such as setting of acquisition time, camera gain, cosmic ray elimination, as well as grating scanning can be performed by WinSpec/32 software installed in the attached PC running under Windows XP. The software can also automatically perform subtraction of background noise from the signal, as well as gluing spectra from two adjacent, overlapping spectral regions to make one spectrum. The spectrum can be directly shown on the PC monitor with the abscissa either in $\mathrm{nm}$, in wavenumbers $\left(\mathrm{cm}^{-1}\right)$ or in relative Raman shifts in wavenumbers.

Initial alignment of the system was performed by observing the fluorescence of a Rhodamine 6G dye placed at the position in the sample and excited by a small CW 532 $\mathrm{nm}$ Nd: YAG laser. The strong fluorescence signal from the dye was visually observed and directed to the interference filter. After reflection from the filter, the signal was visually observed, in sequence, to fall on the mirror M, lens L3 and on the input slit of the spectrograph. Finally, the spectrograph was turned on to observe the fluorescence spectra of the dye (Fig. 2a). The notch centered on $633 \mathrm{~nm}$ on the broadband fluorescence spectrum, is of course, due to the action of the interference filter working in the reflection mode. The rejection ratio of the filter (the ratio of the transmitted intensity on the both sides of the rejection band to the residual transmitted intensity at the center of the blocked band around $633 \mathrm{~nm}$ ) was estimated by performing the necessary measurements on the fluorescence spectrum, taking the background noise into account.

The rejection ratio was found to be about 9. Furthermore, we obtained the notch curve by fitting a smooth fluorescence curve (dotted line) to the data and subtracting the fitted curve from the actual observed notched fluorescence curve. The notch function is shown in Fig. 2b. The width of the notch curve (i.e., FWHM) is approximately $270 \mathrm{~cm}^{-1}$. 


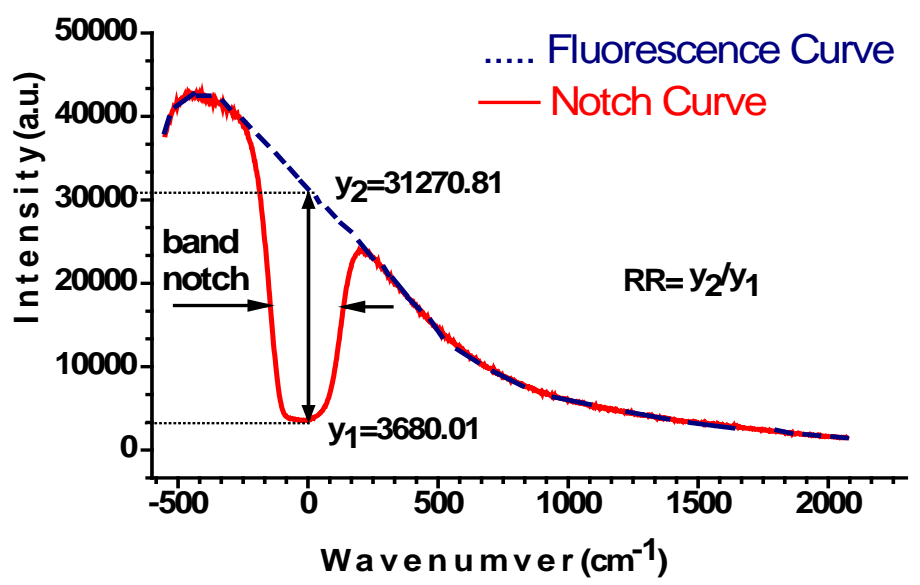

Fig. 2a. Notch performance of the Rayleigh filter in wavenumber shift from the excitation wavelength $(\lambda=632.8 \mathrm{~nm})$ with $1 \mathrm{sec}$. acquisition time.

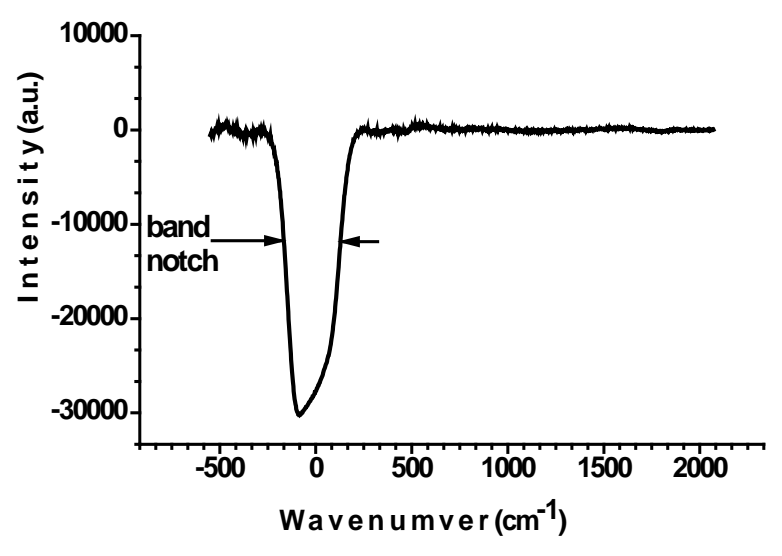

Fig. 2b. Calculated notch function of the interference filter.

\section{RESULTS AND DISCUSSION}

We placed reagent-grade benzene (Merck) in a glass sample bottle and acquired spectra for $60 \mathrm{sec}$. The recorded spectrum, centered on $1500 \mathrm{~cm}^{-1}$, is shown in Fig. 3. The principal vibrational Raman-active line of benzene, resulting from the symmetrical breathing mode of the benzene ring, was observed to have a Raman shift of $993 \mathrm{~cm}^{-1}$. A number of other lines could be clearly seen on both sides of the line in the fingerprint region. The wavenumbers of the principal Raman lines are compared to their literature values in Table 1. The observed Raman lines are in excellent agreement to their literature values within experimental error $\left(<3 \mathrm{~cm}^{-1}\right)$. The ability to capture the relatively weak, minor Raman lines of benzene (e.g., lines at 1179, 609, $850 \mathrm{~cm}^{-1}$ etc.) attests to the sensitivity and efficiency of the constructed laser Raman system. 


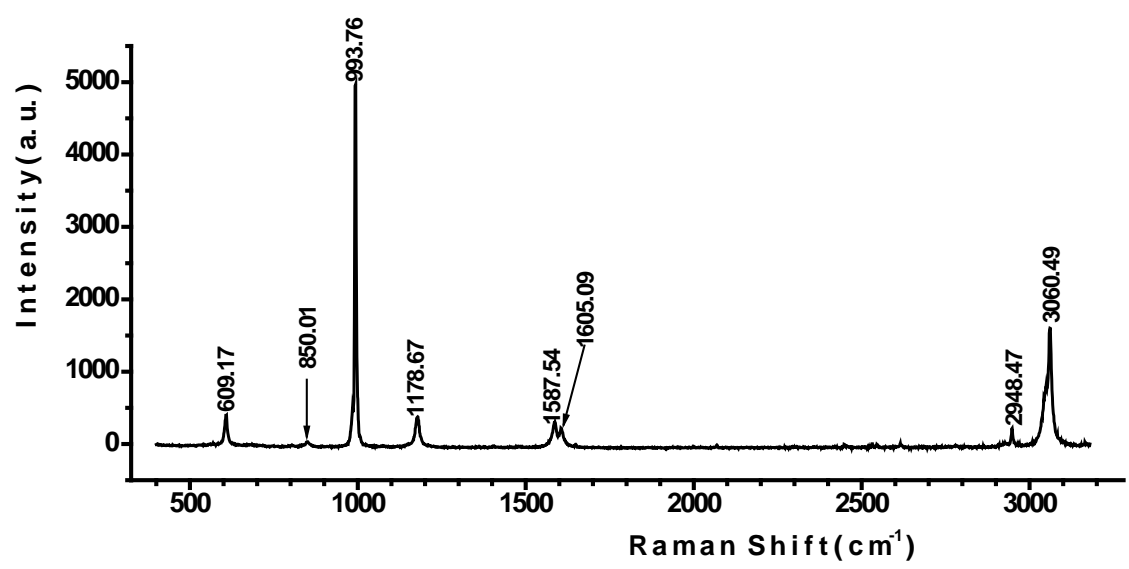

Fig. 3. Raman spectrum of a reagent-grade benzene acquired within 60 sec.

Next, we acquired the Raman spectra of toluene, which is a derivative of benzene. In toluene, due to the presence of the $\mathrm{CH}_{3}$ group, the frequency of the dominant Raman mode was shifted to $1005 \mathrm{~cm}^{-1}$ from $993 \mathrm{~cm}^{-1}$ as compared to benzene. This shift can be used to distinguish toluene from benzene in an unknown sample spectroscopically. The entire glued spectrum of toluene is shown from $200 \mathrm{~cm}^{-1}$ to $3000 \mathrm{~cm}^{-1}$ in Fig. 4.

\section{Table 1}

Comparison between literature values and experimental values of different vibrational modes of benzene

\begin{tabular}{clcc}
\hline Sample & \multicolumn{1}{c}{$\begin{array}{c}\text { Vibrational modes and } \\
\text { functional groups }\end{array}$} & $\begin{array}{c}\text { Literature } \\
\text { values } \\
\left(\mathrm{cm}^{-1}\right)\end{array}$ & $\begin{array}{c}\text { Present } \\
\text { experimental values } \\
\left(\mathrm{cm}^{-1}\right)\end{array}$ \\
\hline $\begin{array}{l}\text { C } \cdots \text { C bonds vibrations in benzene ring } \\
\text { (in plane symmetric stretching) }\end{array}$ & 608 & 609.17 \\
& C-H out-of-plane bending & 851 & 850.01 \\
& Totally symmetric ring breathing & 992 & 993.76 \\
Benzene & C-H in-plane parallel bending & 1178 & 1178.67 \\
& C $\cdots$ C bonds vibrations in benzene ring & 1588 & 1587.54 \\
& $\begin{array}{l}\text { (in plane parallel stretching ) } \\
\text { Symmetric stretching and contraction of }\end{array}$ & $\sim 3047$ & 3049.23 \\
& $\begin{array}{l}\text { C-H bond } \\
\text { Symmetric stretching vibration (inward and } \\
\text { outward) of C-H bond }\end{array}$ & 3061 & 3060.47 \\
\hline
\end{tabular}




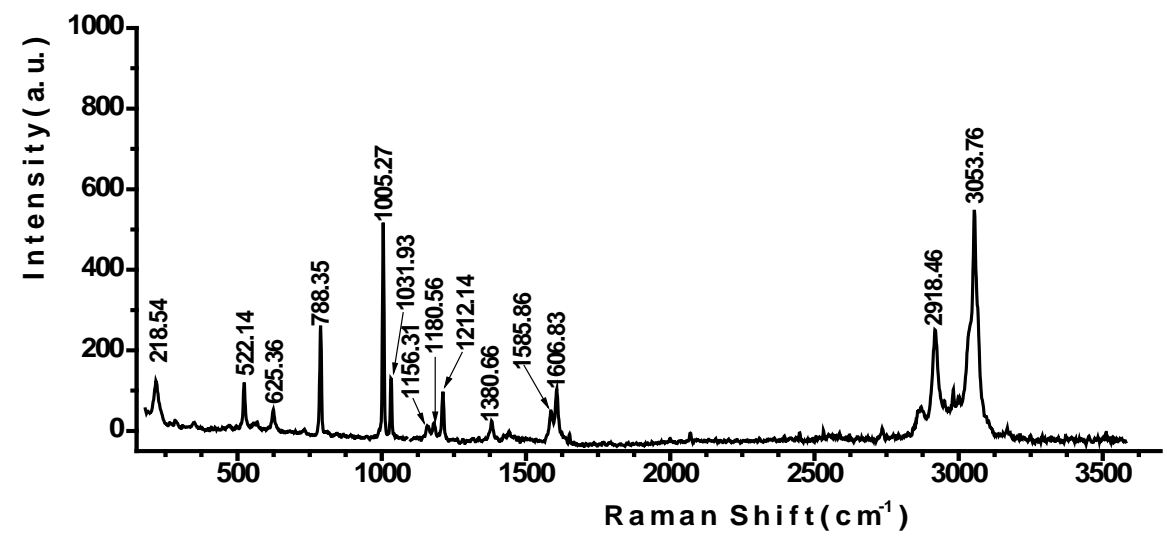

Fig. 4. Raman spectrum of a reagent-grade toluene sample acquired within 60 sec.

Raman spectra of common chemicals such as, ethyl alcohol, glycerine, n-hexane and cyclohexane are shown in Fig. 5. The main features of these spectra are : $883 \mathrm{~cm}^{-1}$ for ethanol (due to C-C-OH skeletal vibration), $1470 \mathrm{~cm}^{-1}$ for glycerine (due to $\mathrm{CH}_{2}$-butterfly mode), $1458 \mathrm{~cm}^{-1}$ for n-hexane (due to $\mathrm{CH}_{2}$-scissors modes), and $1268 \mathrm{~cm}^{-1}$ for cyclohexane (due to $\mathrm{CH}_{2}$-wag vibrations). ${ }^{(10)}$

As a final example, we acquired Raman spectra of carbon tetrachloride $\left(\mathrm{CCl}_{4}\right)$ (Fig. 6a) which has three stokes lines at 219, 316 and $463 \mathrm{~cm}^{-1}$ due to vibration modes of the $\mathrm{CCl}_{4}$ structure. The strongest, $463 \mathrm{~cm}^{-1}$ is due to the totally symmetric vibrations and the other two are due to the $\mathrm{Cl}-\mathrm{C}-\mathrm{Cl}$ bending modes (butterfly modes). These values agree with the corresponding literature values of within $2 \mathrm{~cm}^{-1}$.(2)

In order to observe anti-stokes Raman lines, we blocked the laser, and moved the spectrograph grating to the anti-stokes side of the $633 \mathrm{~nm}$ excitation line. We were able to observe the anti-stokes Raman spectra after a $60 \mathrm{sec}$ acquisition time (Fig. 6b). The lines were of lesser intensity compared to the Raman lines, as expected, and according to the theory of Raman effect, their relative intensity compared to stokes lines is a measure of the temperature of the sample. This also shows that the interference filter has roughly symmetric response on both sides of the laser line, as can be inferred from the calculated notch function in Fig. $2 \mathrm{~b}$. 


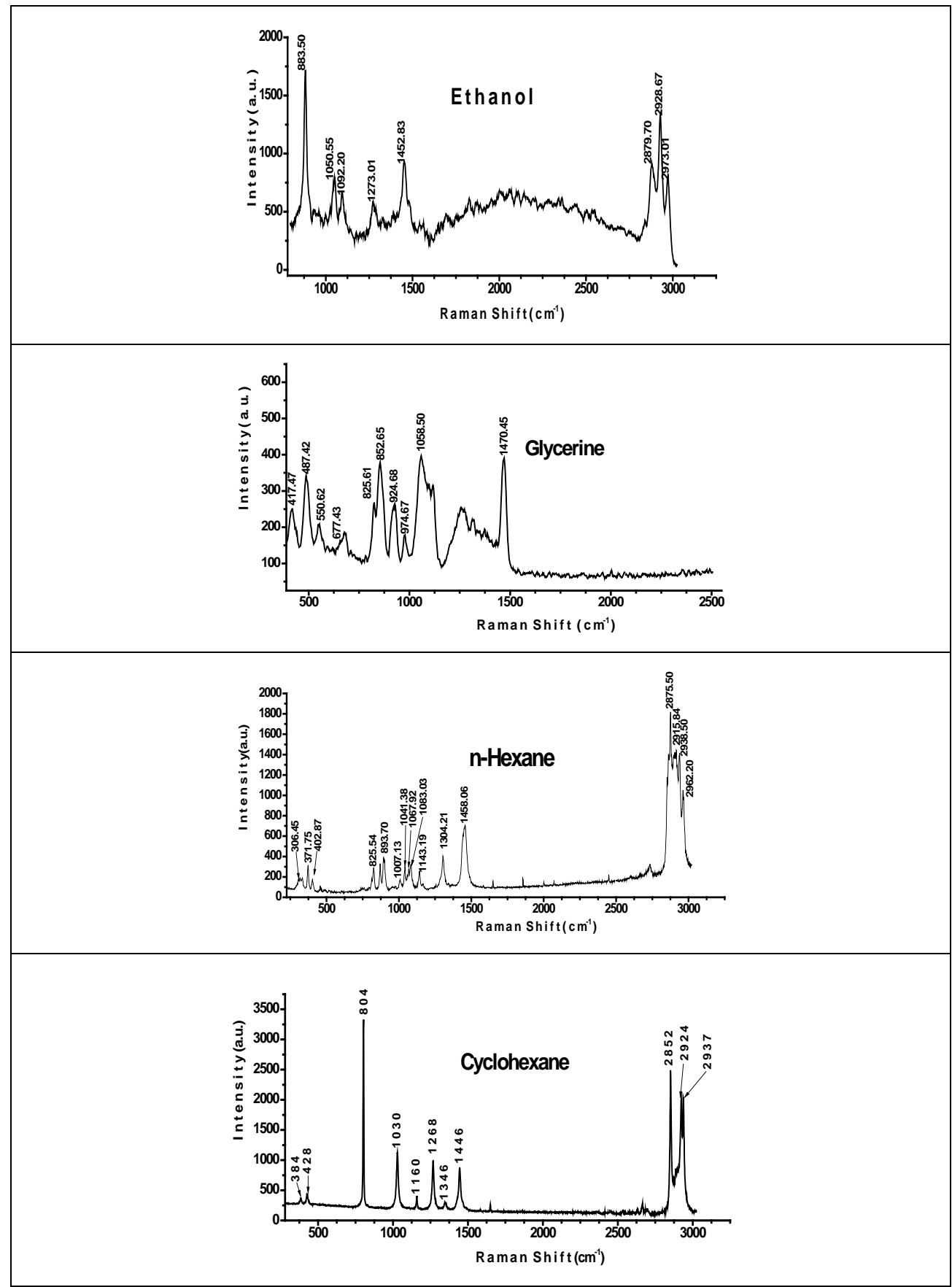

Fig. 5. Raman spectra of some alcohols and aliphatic hydrocarbons acquired in 60 sec. 

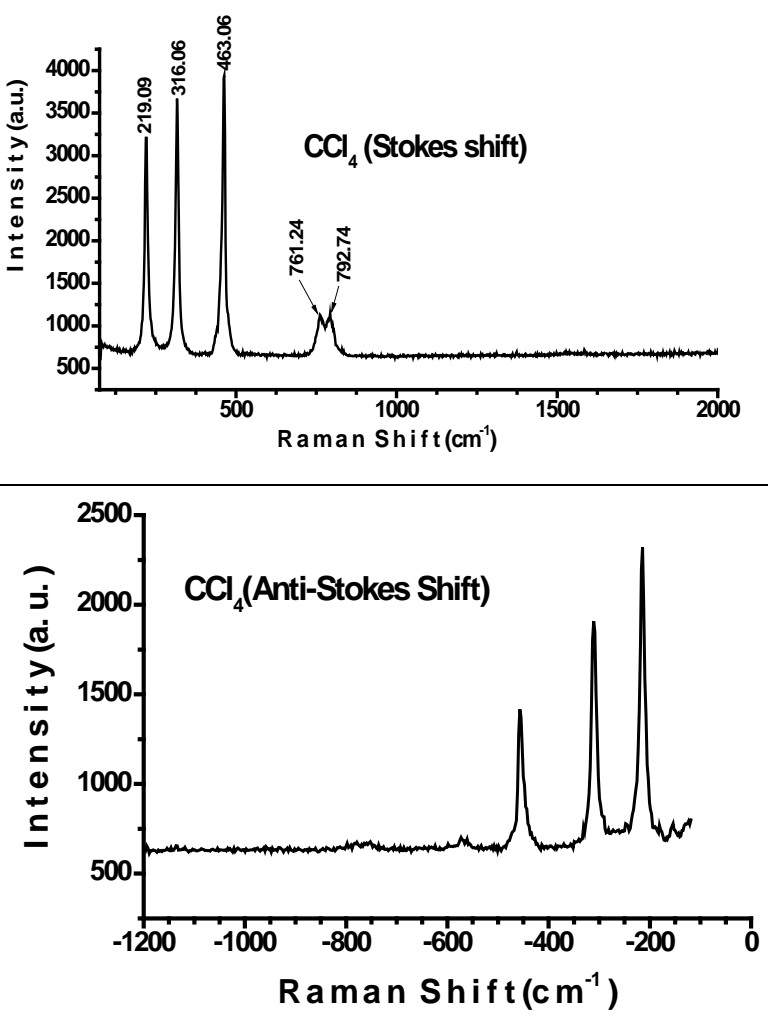

Fig. 6. Stokes (a) and anti-stokes (b) spectra of carbon tetrachloride acquired in 60 sec.

\section{CONCLUSION}

In the present paper, we have shown that it is possible to construct a simple laser Raman system using an interference filter, and commercially available spectrograph and a He-Ne laser. The Raman system was found to have excellent performance and is able to acquire spectra of liquid samples with adequate accuracy and sensitivity. It should be possible to use the system for chemical identification of unknown compounds in a solution. We hope to use the system for a host of new applications, such as determination of composition of petrochemical products, analysis of edible oils and food stuffs, detection of pollutants in surface water, to name a few.

\section{ACKNOWLEDGEMENT}

The work was done using the facilities of the Nonlinear Optics and Laser Spectroscopy Laboratory at the Center of Excellence (COE) of the University of Dhaka. The authors would like to thank Professor A. H. Khan, former Director of the COE, and the current Director of the Center, Professor Syed Saleheen Qadri, for providing them with the facilities of the Center of Excellence.

\section{REFERENCES}

1. C. V. RAMAN AND K. S. KRISHNAN, Nature, 121, 501, 1928. 
2. John R. Ferraro, Kazou Nakamoto and Chris W. Brown, "Introductory Raman Spectroscopy” (2nd ed.), Elsevier Science, 2003.

3. Ewin Smith, Geoffry Dent, "Modern Raman Spectroscopy- A Practical Approach,” Willy, 2005.

4. H.L. Welsh, M.F. Crawford, T.R. Thomas, and G. R. Love, Canadian J. Phys., 30, 577, 1952.

5. S.P.S, PorTO AND D.L. WoOd, J. Opt. Soc. Am., 52, 251, 1962.

6. A. Weber AND S.P.S PorTO, J. Opt. Soc. Am., 55, 1033, 1965.

7. P. E. Schoen AND J. M. Schnur, Applied Spectroscopy, 33, 178, 1979.

8. C. JuliEn, Journal of Optics, 11, 257-267, 1980.

9. CARrabBa, et al. Applied Spectroscopy, 44, 1558-1561, 1990.

10. Norman B. Colthup, LaWrence H. Daly, and Stephen E. Wiberley, "Introduction to Infrared and Raman Spectroscopy”, (2nd ed.), Academic Press, 1975.

Journal of Bangladesh Academy of Sciences, Vol. 32, No. 1, 121-129, 2008 